\title{
A 6-bp deletion in the TYRP1 gene causes the brown colouration phenotype in Chinese indigenous pigs
}

\author{
J Ren, H Mao, Z Zhang, S Xiao, N Ding and L Huang \\ Key Laboratory for Animal Biotechnology of Jiangxi Province and the Ministry of Agriculture of China, Jiangxi Agricultural University, \\ Nanchang, PR China
}

\begin{abstract}
Brown coat colour has been described in Chinese-Tibetan, Kele, and Dahe pigs. Here, we report the identification of a causal mutation underlying the brown colouration. We performed a genome-wide association study (GWAS) on Tibetan and Kele pigs, and found that brown colours in Chinese breeds are controlled by a single locus on pig chromosome 1. By using a haplotype-sharing analysis, we refined the critical region to a $1.5-\mathrm{Mb}$ interval that encompasses only one pigmentation gene: tyrosinaserelated protein 1 (TYRP1). Mutation screens of sequence variants in the coding region of TYRP1 revealed a strong candidate causative mutation (c.1484_1489del).
\end{abstract}

The protein-altering deletion showed complete association with the brown colouration across Chinese-Tibetan, Kele, and Dahe breeds by occurring exclusively in brown pigs $(n=121)$ and lacking in all non-brown-coated pigs $(n=745)$ from 27 different breeds. The findings provide the compelling evidence that brown colours in Chinese indigenous pigs are caused by the same ancestral mutation in TYRP1. To our knowledge, this study gives the first description of GWAS identifying causal mutation for a monogenic trait in the domestic pig.

Heredity (2011) 106, 862-868; doi:10.1038/hdy.2010.129; published online 27 October 2010

Keywords: causal mutation; coat colour; genome-wide association; pig; TYRP1

\section{Introduction}

Coat colour is one of the most strikingly variable and visible traits in mammals, making it a convenient tool for genetic analysis from the beginning of the last century (Searle, 1968; Silvers, 1979). In mammals, the first genetic studies were conducted on coat colour genetics. At present, 368 coat colour loci, including 159 cloned genes, have been described in mice (http://www.espcr.org/ micemut/). The coat colour loci influence the development, differentiation, proliferation, and migration of melanocytes, the construction and transport of melanosomes, as well as the synthesis of melanin. Intriguingly, these colour genes are shared by different species (Jackson, 1994; Barsh, 1996). A notable example is the conserved role of the melanocortin 1 receptor in mammalian pigmentation (Andersson, 2003). Studies of melanocortin 1 receptor have provided valuable insights not only into the biology of pigmentation but also the evolution of domesticated animals (Fang et al., 2009).

The domestic pig displays a bewildering diversity in coat colour, which intrigued scientists for centuries. Two major colour loci and causative mutations have been well characterized in pigs: the KIT gene for the dominant white colour (Johansson Moller et al., 1996; Marklund et al., 1998;

Correspondence: Dr L Huang, Key Laboratory for Animal Biotechnology of Jiangxi Province and the Ministry of Agriculture of China, Jiangxi Agricultural University, Meiling Road, Nanchang 330045, PR China. E-mail: Lushenghuang@hotmail.com

Received 2 June 2010; revised 19 July 2010; accepted 30 July 2010; published online 27 October 2010
Giuffra et al., 2002; Pielberg et al., 2002) and the MCIR locus for coloured phenotypes (Kijas et al., 1998, 2001). For other colour phenotypes, such as belt (Giuffra et al., 1999) and black-and-tan (Drögemüller et al., 2006) in Western pigs, the causative mutation(s) has not yet been found.

Selective breeding during the long period has resulted in diverse coat colour phenotypes in Chinese indigenous breeds, including solid black, white (Lai et al., 2007), brown (Mao et al., 2010), belted and spotted ( $\mathrm{Xu}$ et al., 2006) pigment types. In most breeds, all pigs share the same colour pattern as breed character. However, variable colours are allowed within the standard in a few breeds, such as Tibetan, Kele, and Dahe pigs (Zhang et al., 1986).

Tibetan pigs, renowned for their unique adaptability to the adverse living conditions of the Qinghai-Tibetan plateau, segregate for black, reddish, and silver brown colours (Figure 1). The colour variants fit an autosomal monogenic model of inheritance and the brown colour is a codominant trait. Heterozygous $(B / b)$ animals display the reddish brown phenotype and homozygotes show silver brown $(b / b)$, or black colours $(B / B)$ (Zhang et al., 1986). Kele and Dahe pigs are two local populations from the Yunnan-Guizhou plateau classified as a single breed (namely, Wujin). A range of light to dark brown shades, as well as a black phenotype (Figure 1), has been observed in Kele and Dahe pigs. Different brown shades are caused by epistatic interaction between two loci (Lian and $\mathrm{Wu}, 1985)$. So far, the molecular basis of the brown phenotypes of Tibetan, Kele, and Dahe pigs remains elusive. Here, we report a genome-wide association study (GWAS) and haplotype-sharing analyses that 

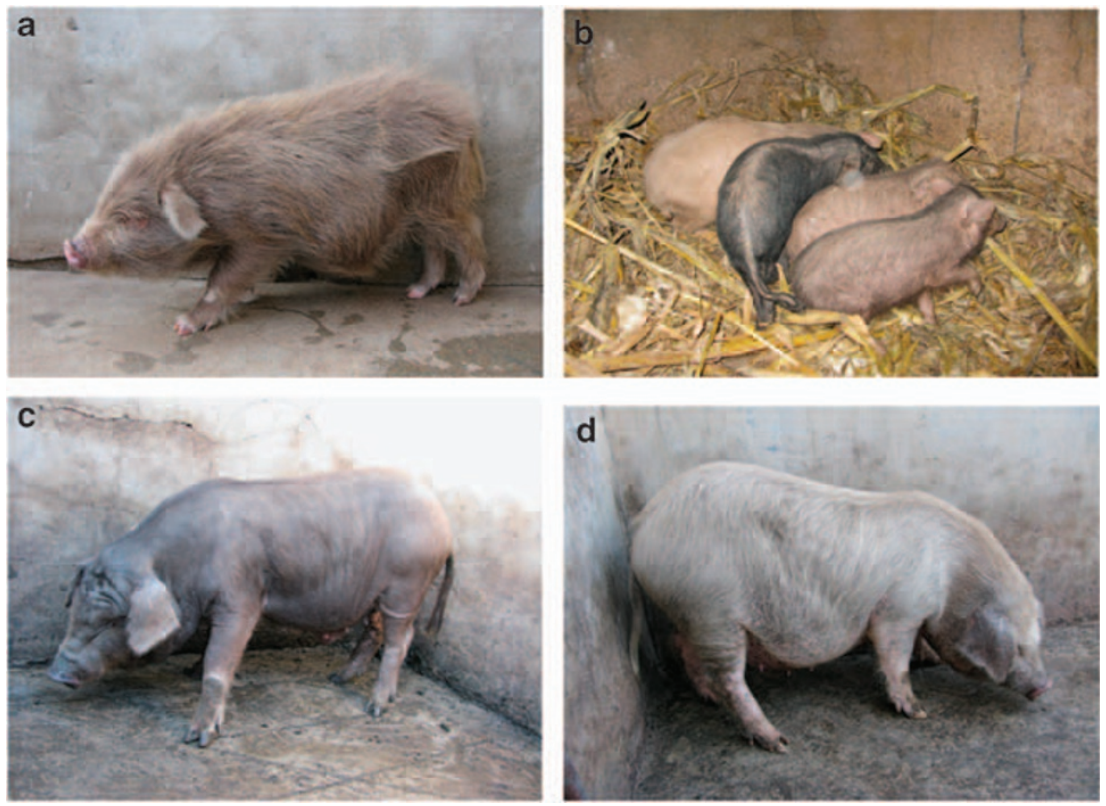

Figure 1 Brown coat colour phenotypes in Chinese indigenous pigs. (a) A reddish brown Tibetan piglet. (b) A flock of Kele piglets with dark brown, pale brown, and black colouration phenotypes. (c) A black brown Dahe sow. (d) A silver brown Dahe sow.

characterize the responsible gene and subsequent detection of a genetic variant that appears to cause the brown colouration in Chinese breeds.

\section{Materials and methods}

\section{Sources of animal samples}

Tibetan pigs were sampled from a multiplying population with brown colour variants in Guangdong province $(n=127)$ and a nucleus herd with the solid black phenotype in the Gongbujiangda county of the Tibetan Autonomous Region $(n=32)$. Brown Tibetan pigs are descendants of one brown boar and two brown sows from the Gannan Tibetan Autonomous Prefecture of Gansu Province. A total of 65 Tibetan pigs, including 32 reddish brown, 3 silver brown, and 30 black individuals from the multiplying population, were used for the genome-wide association mapping. Of the 65 pigs, 32 individuals from 4 families, clearly segregating for the brown phenotype (Supplementary Figure S1), were used for a genome-wide linkage analysis.

Black and brown-coated Kele pigs $(n=86)$ were collected from the Hezhang county of Guizhou province. Of the 86 pigs, 48 pigs from 8 dam families, including 24 dark brown, 2 pale brown, and 22 black individuals, were used for the genome-wide association mapping. Brown-coated Dahe pigs $(n=36)$ were sampled from the Fuyuan county of Guizhou province. A panel of 966 pigs from 27 breeds and wild boars representing a range of distinct coat colours (Table 1) were used for validation of candidate causative mutations. Individual phenotypes of Tibetan, Kele, and Dahe pigs were determined by direct visual inspection and photographs taken outdoors on a sunny day. Coat colour phenotypes of the remaining breeds were obtained directly from the breed registration association. Genomic DNA of all samples was extracted from ear tissues according to standard protocols.

\section{Genome-wide association analysis and haplotype reconstruction}

The PorcineSNP60 BeadChips (Illumina, San Diego, CA, USA) were used to genotype the 65 Tibetan and 48 Kele pigs on an Illumina iScan System following the manufacturer's protocol. Bead arrays with call rate $<85 \%$ were excluded for further analyses. GWASs were performed in a case-control way under the dominant model on all single-nucleotide polymorphisms (SNPs) with a minor allele frequency $>0.05$ and call rate $>95 \%$ by using PLINK (http://pngu.mgh.harvard.edu/purcell/ plink/). The genome-wide significance was calculated by phenotype permutation testing $(n=100000)$. A genome-wide linkage analysis was performed using CRIMAP (available at http://linkage.rockefeller.edu/ soft/crimap/). We assumed that the brown phenotype was inherited as a biallelic autosomal codominant trait with complete penetrance. Faithful haplotypes in the mapped interval were reconstructed with DualPHASE (Druet and Georges, 2010). The most likely responsible gene (TYRP1) was identified by the pig-human comparative map on the basis of the well-characterized coat colour genes.

\section{TYRP1 sequencing}

To detect genetic variants in the coding region of the TYRP1 gene, we designed six intronic primer pairs flanking TYRP1 exons 2-7 and one primer pair for the $3^{\prime}$ UTR from publicly available sequences (NM_001025226 and NC_010443.1). Primer sequences are listed in Supplementary Table S1. Genomic DNA of 5 black and 6 brown Tibetan pigs, 6 Chinese and European wild boars, 48 pigs from 16 different breeds were amplified with these primers. Amplifications were performed in a $25-\mu 1$ reaction containing 0.4 units Taq DNA polymerase (TaKaRa, Dalian, China) and a magnesium concentration of $2.0 \mathrm{mM}$ at optimal annealing temperatures (Supplementary Table S1) using standard protocols. PCR 
Table 1 Genotype and allele frequencies of the TYRP1 c.1484_1489del (p.M495_G496del) mutation in Chinese and European pig breeds

\begin{tabular}{|c|c|c|c|c|c|c|}
\hline \multirow[t]{2}{*}{ Breed } & \multirow[t]{2}{*}{ Coat colour } & \multirow[t]{2}{*}{ No. } & \multicolumn{3}{|c|}{ Genotype } & \multirow{2}{*}{$\begin{array}{l}\text { The del } \\
\text { frequency }\end{array}$} \\
\hline & & & $+/+$ & $+/$ del & del/del & \\
\hline \multicolumn{7}{|l|}{ Chinese breed } \\
\hline \multirow[t]{3}{*}{ Tibetan } & Reddish brown & 51 & 0 & 51 & 0 & 0.50 \\
\hline & Silver brown & 5 & 0 & 0 & 5 & 1.00 \\
\hline & Black & 103 & 103 & 0 & 0 & 0.00 \\
\hline \multirow[t]{3}{*}{ Kele } & Dark brown & 27 & 0 & 27 & 0 & 0.50 \\
\hline & Pale brown & 2 & 0 & 0 & 2 & 1.00 \\
\hline & Black & 57 & 57 & 0 & 0 & 0.00 \\
\hline \multirow{3}{*}{ Dahe } & Black brown & 10 & 0 & 10 & 0 & 0.50 \\
\hline & Yellow brown & 21 & 0 & 21 & 0 & 0.50 \\
\hline & Silver brown & 5 & 0 & 0 & 5 & 1.00 \\
\hline Dianan small ear & Black & 31 & 31 & 0 & 0 & 0.00 \\
\hline Erhualian & Black & 31 & 31 & 0 & 0 & 0.00 \\
\hline Hetao large ear & Black & 32 & 32 & 0 & 0 & 0.00 \\
\hline Jinhua & Two-end-black & 33 & 33 & 0 & 0 & 0.00 \\
\hline Laiwu & Black & 32 & 32 & 0 & 0 & 0.00 \\
\hline Luchuan & Black with white belly & 34 & 34 & 0 & 0 & 0.00 \\
\hline New Dahe & Black & 44 & 44 & 0 & 0 & 0.00 \\
\hline Rongchang & White & 34 & 34 & 0 & 0 & 0.00 \\
\hline Yushan & Black & 31 & 31 & 0 & 0 & 0.00 \\
\hline Wuzhishan & Black with white belly & 32 & 32 & 0 & 0 & 0.00 \\
\hline Wild boars & Wild type & 72 & 72 & 0 & 0 & 0.00 \\
\hline \multicolumn{7}{|l|}{ European breed } \\
\hline Berkshire & Black & 4 & 4 & 0 & 0 & 0.00 \\
\hline British Lop & White & 1 & 1 & 0 & 0 & 0.00 \\
\hline Chester White & White & 2 & 2 & 0 & 0 & 0.00 \\
\hline Duroc & Red & 67 & 67 & 0 & 0 & 0.00 \\
\hline Hampshire & White belt and black body & 42 & 42 & 0 & 0 & 0.00 \\
\hline Iberian & Black & 10 & 10 & 0 & 0 & 0.00 \\
\hline Landrace & White & 39 & 39 & 0 & 0 & 0.00 \\
\hline Large Black & Black & 2 & 2 & 0 & 0 & 0.00 \\
\hline Large White & White & 64 & 64 & 0 & 0 & 0.00 \\
\hline Mid White & White & 2 & 2 & 0 & 0 & 0.00 \\
\hline Old Spot & Spotted & 2 & 2 & 0 & 0 & 0.00 \\
\hline Pietrain & Spotted & 27 & 27 & 0 & 0 & 0.00 \\
\hline Saddle Back & White belt and black body & 1 & 1 & 0 & 0 & 0.00 \\
\hline Tamworth & Tan and black & 1 & 1 & 0 & 0 & 0.00 \\
\hline Yorkshire & White & 2 & 2 & 0 & 0 & 0.00 \\
\hline Wild boars & Wild type & 13 & 13 & 0 & 0 & 0.00 \\
\hline
\end{tabular}

products were bidirectionally sequenced with original PCR primers. Sequence data were assembled and aligned with ClustalX (http://abs.cit.nih.gov/clustalx/) to reveal genetic variants.

\section{Mutation genotyping}

A KpnI PCR-RFLP assay was used to genotype the TYRP1 c.1484 1489del mutation. Genomic DNA was amplified with primers for TYRP1 exon 8 (Supplementary Table S1) as described above. PCR products were digested with KpnI (New England Biolabs, Ipswich, MA, USA) at $37^{\circ} \mathrm{C}$ for $4 \mathrm{~h}$. The restriction fragments were separated on $2 \%$ agarose gels and genotypes were determined from the resulting band patterns. For genotyping the c.428G $>$ A polymorphism, primers for TYRP1 exon 3 (Supplementary Table S1) were used to amplify a 736-bp fragment containing the mutation site. The resulting PCR products were subjected to $\mathrm{SNaPshot}$ analyses with an extension primer $\left(5^{\prime}\right.$-TTTTTTTTT TTTTACCTAAGTACAGAAGAAAAGAACC- $\left.3^{\prime}\right)$ and the ABI PRISM SNaPshot Multiplex Kit (Applied Biosystems, Foster City, CA, USA) according to the manufacturer's protocol. SNaPshot products $(0.5 \mu \mathrm{l})$ were mixed with $9 \mu \mathrm{l}$ of formamide and $0.5 \mu \mathrm{l}$ of GeneScan-120 LIZ Size Standard and separated by capillary electrophoresis in a 3130XL Genetic Analyzer (Applied Biosystems). The genotype data were finally recorded with GeneMapper 4.0 software (Applied Biosystems).

\section{Results}

\section{Mapping of the responsible gene}

Of the 62163 SNPs on BeadChip (Ramos et al., 2009), 48174 SNPs in Tibetan samples and 46508 SNPs in Kele pigs had a call rate $>95 \%$ and had annotated genomic positions on Build 7.0 of the Sus scrofa genome (http:// www.sanger.ac.uk/Projects/S_scrofa/). Four Tibetan pigs having an overall call rate $<85 \%$ were discarded for further analyses. A final panel of 30342 and 41347 informative SNPs with minor allele frequency $>0.05$ was included in the GWAS on 61 and 48 successfully genotyped Tibetan and Kele pigs, respectively. The 30342 SNPs were also used for a genome-wide linkage mapping analyses in four Tibetan families.

In Tibetan pigs, the GWAS identified the most strongly associated SNP (INRA0006065, nominal $P$-value= 

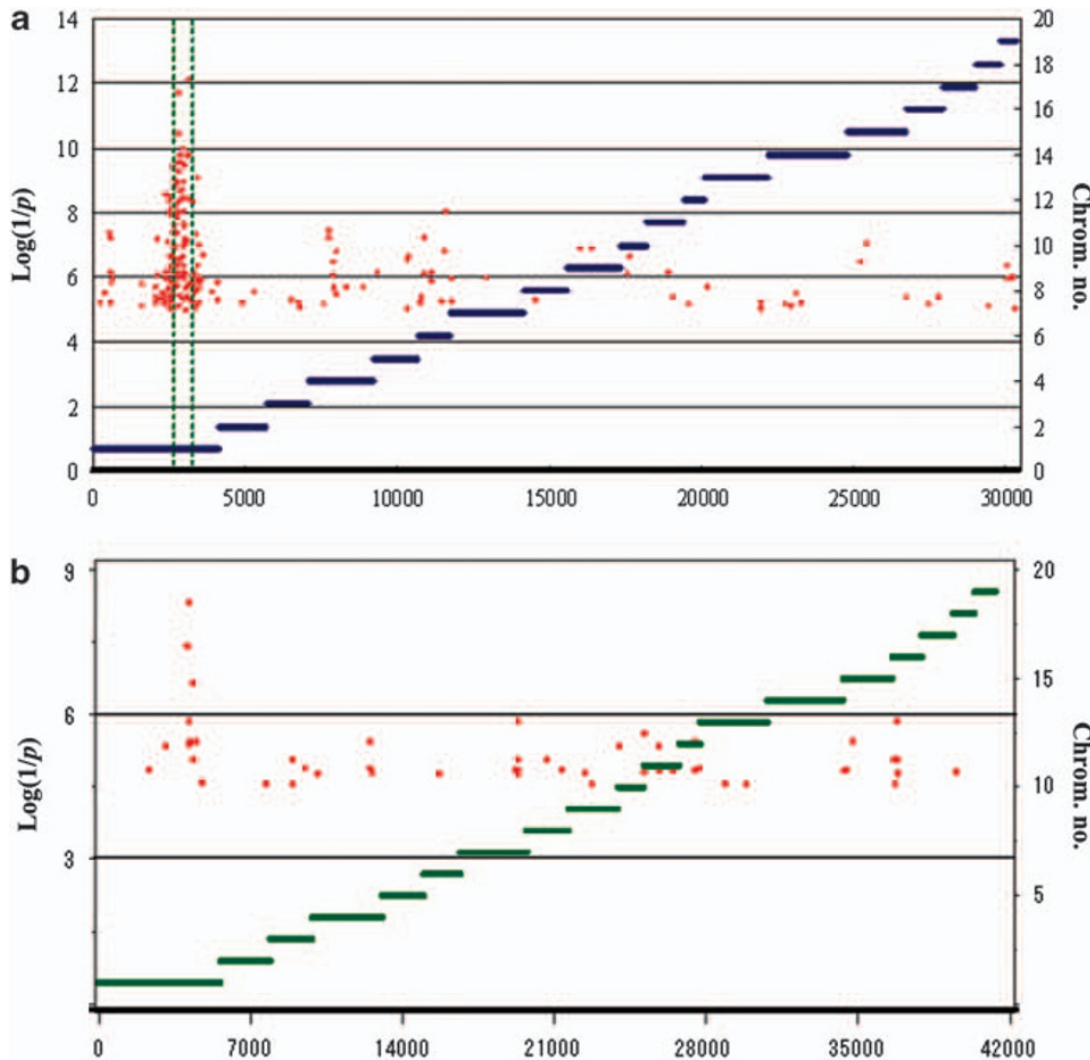

Figure 2 Genome-wide association mapping of the brown coat colour locus in Tibetan (a) and Kele (b) pigs. The black horizontal bars indicate the limits between the 18 autosomes. Evidence for linkage ( $y$ axis) is measured as $\log (1 / p)$. Only genome-wide significant markers are shown. The genome-wide significant threshold was determined by 100000 phenotype permutations. The green vertical dashed line represents the non-recombinant region in four Tibetan families.

$7.3 \times 10^{-13}$ ) at $194.62 \mathrm{Mb}$ on chromosome 1 (SSC1), followed by SNP (INRA0005584, nominal $P$-value $=$ $1.9 \times 10^{-12}$ ) at $165.46 \mathrm{Mb}$ on this chromosome. This association is 100-fold stronger than that for any other region in the genome (Figure 2a). By using linkage analyses on four Tibetan families, we observed that three SNPs (INRA0005874 at $182.55 \mathrm{Mb}$, ALGA0007793 at $184.00 \mathrm{Mb}$, and INRA0005944 at $188.64 \mathrm{Mb})$ in the INRA0006065-INRA0005584 region $(\sim 29 \mathrm{Mb})$ showed complete linkage disequilibrium with the brown locus. These observations strongly support that the responsible gene locates in the 29-Mb region on SSC1.

In Kele pigs, SNP INRA0005944 at $188.64 \mathrm{Mb}$ on SSC1 showed the strongest association (nominal Pvalue $\left.=4.6 \times 10^{-9}\right)$. SNPs ALGA0007745 at $182.29 \mathrm{Mb}$ and DRGA0002004 at $196.38 \mathrm{Mb}$ on SSC1 define the critical interval $(\sim 14 \mathrm{Mb})$, as the association of SNPs in the interval is 100-fold stronger than that for any other region in the genome (Figure $2 b$ ). The mapped interval perfectly overlaps the $29-\mathrm{Mb}$ region harbouring the Tibetan brown locus. This observation supports the assumption that a common mutation underlies the brown phenotypes in Chinese indigenous pigs. We, hence, reconstructed haplotypes around the mapped interval for all tested animals. A $1.5-\mathrm{Mb}$ region from 183.1 to $184.6 \mathrm{Mb}$ on SSC1 appears to be the crucial interval, as it defines the minimum shared haplotype among brown chromosomes from Tibetan and Kele pigs (Figure 3). We conclude that the causal mutation is located in this interval.
As the quality of the pig genome annotation is still far from perfect, we inferred the gene annotation of the critical interval from the corresponding human region. The human orthologous region on HSA9 (assembly GRCh37) contains only three annotated genes, including one well-characterized pigmentation gene: tyrosinaserelated protein 1 (TYRP1), which is at $\sim 200 \mathrm{~kb}$ from the homologous sequence of the SNP ALGA0007793 in the $1.5-\mathrm{Mb}$ critical interval. In humans, TYRP1 mutations lead to oculocutaneous albinism type 3, known as Brown and Rufous OCA (Sarangarajan and Boissy, 2001). Moreover, TYRP1 plays a well-established and crucial role in mammalian pigmentation and refers to the classic brown locus in mice (Jackson et al., 1990; Zdarsky et al., 1990; Javerzat and Jackson, 1998). Mutations in TYRP1 are repeatedly associated with brownish coat colour in a number of domestic animals, including cat (Lyons et al., 2005; Schmidt-Kuntzel et al., 2005), dog (Schmutz et al., 2002), cattle (Berryere et al., 2003), and sheep (Gratten et al., 2007), as well as quail (Nadeau et al., 2007) and fish (Braasch et al., 2009). We, therefore, selected TYRP1 as the prime candidate gene and investigated whether mutations in TYRP1 might be responsible for the brown phenotypes in Chinese pig breeds.

\section{Mutations in TYRP1}

We designed PCR primers (Supplementary Table S1) to amplify all seven protein-coding exons and exon-intron boundary sites of TYRP1. Mutation screening of 5 black 


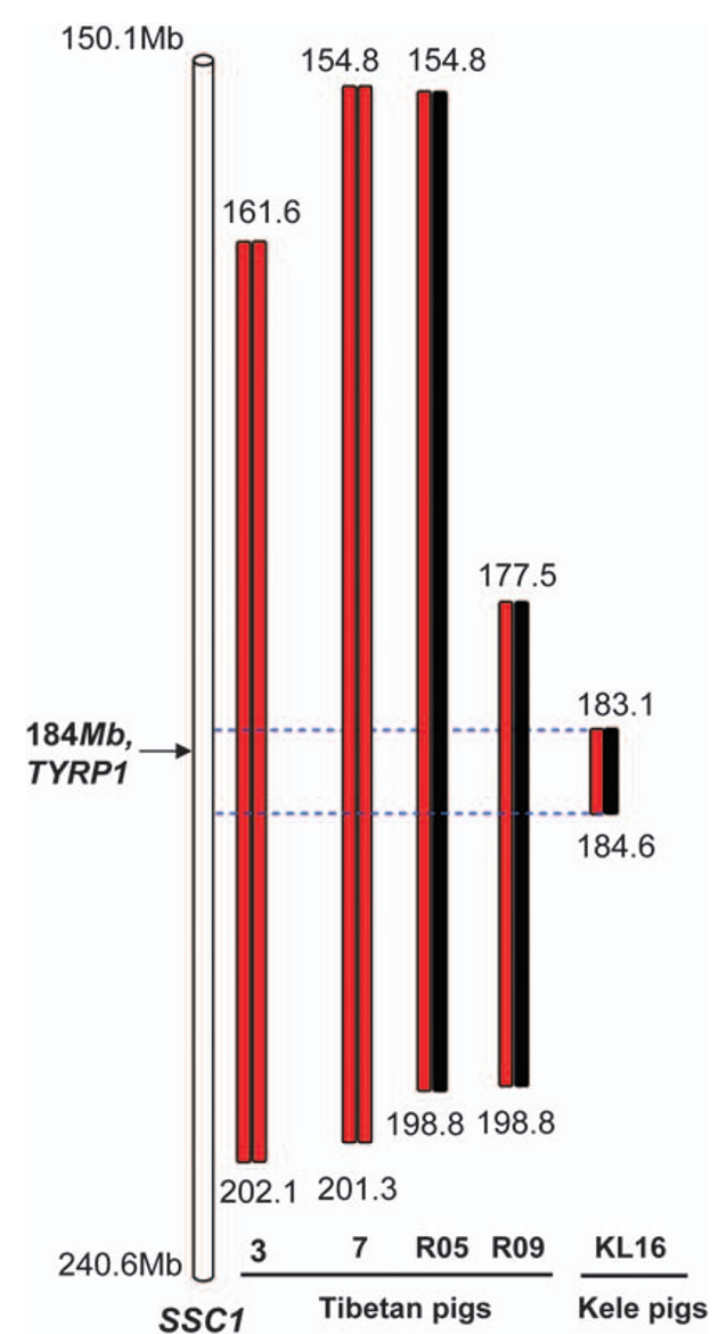

Figure 3 Shared haplotypes in the critical region harbouring the brown locus between Tibetan and Kele pigs. Red bars indicate the brown haplotype and black bars represent the non-brown haplotype. Only haplotypes of five individuals faithfully reconstructed by the hidden Markov model (DualPHASE) on the basis of family information are shown. The boundaries of homozygous haplotype blocks are given in $\mathrm{Mb}$. The Sus scrofa genome assembly (Build 7.0, available at http://www.ensembl.org/Sus_scrofa/Info/Index) is used as standard reference. The horizontal dashed green lines indicate the minimum shared haplotype block of $1.5 \mathrm{Mb}$.

and 6 brown Tibetan samples, as well as 54 animals from 18 breeds, revealed 22 TYRP1 sequence variants. Of the 22 polymorphisms, 13 SNPs were located in introns and none altered the conserved splice sites. The intronic SNPs occurred in both brown-coloured and non-browncoated breeds, and were therefore not analysed further. Of the nine mutations within the coding region, seven substitutions also segregated in both brown-coloured and non-brown-coated breeds (Supplementary Table S2), and were apparently excluded as the causative mutation. Two other coding variants result in amino acid changes: c.428G > A and c.1484_1489delTGGGTA (Supplementary Figure S3). The non-synonymous c.428G > A polymorphism at exon 2 leads to a transition from a histidine to arginine at amino acid 143 of the protein (p.143His $>$ Arg). The 6-bp deletion in exon 8, c.1484_1489del, removes two amino acids from protein at positions 495 and 496 (p.M495_G496del), respectively. We performed alignment of the TYRP1 protein sequence from 13 vertebrate species and found that the hexanucleotide deletion occurs in a conserved transmembrane domain region predicted by SMART (http://smart. embl-heidelberg.de/) (Supplementary Figure S3). The two exonic mutations are likely of functional significance and were hence analysed further. We used KpnI PCRRFLP to genotype the deletion polymorphism using primers for exon 8 (Supplementary Table S1). The wildtype allele is represented by two fragments of 398 and $347 \mathrm{bp}$ and the del allele by an uncut amplicon of $745 \mathrm{bp}$ (Supplementary Figure S2). The c.428G > A polymorphism was genotyped by a SNaPshot assay (Supplementary Figure S2).

Association of TYRP1 mutations with brown phenotypes We investigated the distribution of genotypes at the c.428G > A and c.1484_1489del sites in 966 pigs from 27 different breeds and wild pigs, representing a wide range of colour phenotypes. The analysis revealed that a majority of the non-brown-coated breeds segregated for the c.428G > A polymorphism (Supplementary Table S3). This excluded this missense mutation as the cause of the brown coat colour phenotype. Intriguingly, we found a perfect association of the c.1484_1489del mutation with the brown phenotype. All 121 brown-coated pigs from Tibetan, Kele, and Dahe pigs were homozygous or heterozygous for the del allele (Table 1). None of the 160 black Tibetan and Kele pigs, and 685 pigs from other 27 diverse pig breeds and wild boars had the del allele ( $\chi^{2}$-test, $P=4.41 \times 10^{-212}$; Table 1$)$. In Tibetan pigs, all the presumed heterozygous animals in the family samples (Supplementary Figure S1) carried the +/del genotype. Moreover, all heterozygotes had the reddish brown phenotype, whereas the del/del animals uniformly displayed the silver brown colouration, reflecting that this mutation perfectly fitted the proposed codominant model of inheritance for the phenotype. Kele and Dahe animals homozygous for the del allele show pale or silver brown colouration, indistinguishable from those observed in Tibetan pigs. However, discordant brown shades are observed among $+/$ del animals of Tibetan (reddish brown), Kele (dark brown), and Dahe (black or yellow brown) pigs.

\section{Discussion}

Genome-wide association analysis on the basis of largescale SNP scan is emerging as a new and powerful approach for high resolution genetic analysis in domestic animals (Andersson, 2009), but to our knowledge this approach has not yet been reported in pigs. In this study, we used newly developed PorcineSNP60 BeadChip to perform a genome scan for the brown coat colour locus in Chinese pig breeds. The majority of SNPs on BeadChip were originally discovered from western commercial breeds and wild pigs (Ramos et al., 2009). Applications of the chip in Asian breeds are hence cautioned for the ascertainment bias. Nevertheless, 64.1 and $66.5 \%$ of the SNPs on the chip are segregating in the Tibetan and Kele breeds with minor allele frequency $>0.05$, proving the usefulness of BeadChip in Chinese pig populations, although not as powerful as the chip did in western breeds. 
The GWAS identified significantly associated regions in Tibetan and Kele pigs that perfectly overlap on SSC1, indicating that the brown mutation in the tested breeds is derived from a common ancestor. The mapped intervals are unexpectedly large $(\sim 29$ and $14 \mathrm{Mb})$. This is most likely caused by very strong linkage disequilibrium in tested animals. Chinese local breeds, including Tibetan, Kele, and Dahe pigs, have been endangered and their number and size have decreased dramatically in recent years resulting from the wide introduction of international breeds for commercial interests. A high level of inbreeding is hence expected to occur in these threatened breeds, leading to strong linkage disequilibrium and large mapped intervals in the GWAS.

Haplotype-sharing analysis further revealed a critical region of $1.5 \mathrm{Mb}$ on SSC1 that encompasses only one pigmentation gene, TYRP1. We, therefore, investigated TYRP1 variants in a collection of diverse pig breeds and identified a strong candidate causative mutation: c.1484_1489del. The deletion causes a lack of two amino acids in a predicted transmembrane domain of the protein and is likely of functional significance. Of the TYRP1 functional variants affecting brown phenotypes in vertebrates, none affect the two amino acids. The protein-altering deletion showed complete association with the brown colouration across Tibetan, Kele, and Dahe breeds by occurring exclusively in brown pigs and lacking in all non-brown-coated pigs from 27 different breeds. The perfect association across breeds, together with high-resolution mapping of the responsible locus, positional characterization of the strong candidate gene, protein-altering nature, and concordant inheritance model of the mutation, collectively provide evidence of the casualty of TYRP1 c.1484_1489del for the brown coat colour phenotypes in Chinese breeds.

$T Y R P 1$ functional mutations usually show a recessive inheritance in vertebrates except for a dominant mutation causing the white-based brown in mice (Zdarsky et al., 1990). Interestingly, the c.1484_1489del mutation is inherited in a codominant way. One explanation is that the mutation is a dominant-negative mutation, which interferes with the wild allele and consequently leads to a different phenotype in the heterozygote. Alternatively, the brown phenotype might be caused by TYRP1 haploinsufficiency in Chinese pigs.

The causative mutation is probably of Asian origin and occurred after domestication, as determined by its absence in European breeds and wild pigs. It is not a recent mutation as brown-coated Dahe and Kele pigs have been reported as early as the seventeenth century (Zhang et al., 1986). Black Tibetan pigs and dark brown Kele pigs have always fascinated their human owners, whereas yellow brown-coated Dahe pigs are generally favoured compared with silver brown individuals (Lian and $\mathrm{Wu}, 1985)$. A molecular test for the c.1484_1489del mutation will give breeders an efficient and accurate tool to breed for brown phenotypes or to select against them.

More than a single TYRP1 mutation have been associated with various brown shades in mice (Jackson et al., 1990; Zdarsky et al., 1990; Javerzat and Jackson, 1998), cats (Lyons et al., 2005; Schmidt-Kuntzel et al., 2005), and dogs (Schmutz et al., 2002). We hence expected TYRP1 allele series for brown variants in Chinese pigs. Nevertheless, we did not identify other equivalently significant variants in the coding region of TYRP1 gene except for the common causative variant, c.1484_1489del. There are several possible explanations. The first is the involvement of epistatic effect of other coat colour genes. The interaction between TYRP1 and melanocortin 1 receptor has been shown to be important in pigmentation in dogs (Schmutz et al., 2002). However, we found that the epistasis interaction for TYRP1 and melanocortin 1 receptor did not explain the brown shades in Kele and Dahe pigs (Supplementary Table S4). A further investigation on other pigmentation genes, including dilution genes, is worthwhile. The second possible explanation is minor polygenic or modifier gene effects leading to variations in pigment intensity.

In conclusion, we have identified the c.1484_1489del mutation in the TYRP1 gene as the causative mutation for the brown colouration in Chinese indigenous pigs. This result provides the first direct evidence for the involvement of TYRP1 in pigmentation of pigs and advances the understanding of the molecular basis of colour variation in pigs. Our study also provides a genetic testing tool to breed for or against brown coat colour in Chinese indigenous pigs.

\section{Accession numbers}

The coding sequences of TYRP1 determined in this study have been submitted to GenBank with accession numbers GU345783-GU345801.

\section{Conflict of interest}

The authors declare no conflict of interest.

\section{Acknowledgements}

The authors would like to thank Zhizhong Li (the brown Tibetan pig owner), Professor Linsheng Lian (Yunnan Agricultural University) and Dr Junxun Wang (the Ministry of Agriculture of China) for their kind help in sample collection. The authors also thank Dr Carole Charlier at the University of Liège for valuable comments on positional candidate genes and Dr Leif Andersson at Uppsala University for critical comments on the manuscript. This work was funded by grants from National Natural Science Foundation of China (30425045 and 30871771) to Lusheng Huang and Jun Ren.

\section{References}

Andersson L (2003). Melanocortin receptor variants with phenotypic effects in horse, pig, and chicken. Ann NY Acad Sci 994: 313-318.

Andersson L (2009). Genome-wide association analysis in domestic animals: a powerful approach for genetic dissection of trait loci. Genetica 136: 341-349.

Barsh GS (1996). The genetics of pigmentation: from fancy genes to complex traits. Trends Genet 12: 299-305.

Berryere TG, Schmutz SM, Schimpf RJ, Cowan CM, Potter J (2003). TYRP1 is associated with dun coat colour in Dexter cattle or how now brown cow? Anim Genet 34: 169-175.

Braasch I, Liedtke D, Volff J-N, Schartl M (2009). Pigmentary function and evolution of tyrp1 gene duplicates in fish. Pigment Cell Melanoma Res 22: 839-850.

Drögemüller C, Giese A, Martins-Wess F, Wiedemann S, Andersson L, Brenig B et al. (2006). The mutation causing the black-and-tan pigmentation phenotype of Mangalitza 
pigs maps to the porcine ASIP locus but does not affect its coding sequence. Mamm Genome 17: 58-66.

Druet T, Georges M (2010). A hidden markov model combining linkage and linkage disequilibrium information for haplotype reconstruction and QTL fine mapping. Genetics 184: 789-798.

Fang MY, Larson G, Ribeiro HS, Li N, Andersson L (2009). Contrasting mode of evolution at a coat color locus in wild and domestic pigs. PLoS Genet 5: e1000341.

Giuffra E, Evans G, Tornsten A, Wales R, Day A, Looft H et al. (1999). The Belt mutation in pigs is an allele at the Dominant White (I/KIT) locus. Mamm Genome 10: 1132-1136.

Giuffra E, Törnsten A, Marklund S, Bongcam-Rudloff E, Chardon P, Kijas JMH et al. (2002). A large duplication associated with Dominant White color in pigs originated by homologous recombination between LINE elements flanking KIT. Mamm Genome 13: 569-577.

Gratten J, Beraldi D, Lowder BV, Mcrae AF, Visscher PM, Pemberton JM et al. (2007). Compelling evidence that a single nucleotide substitution in TYRP1 is responsible for coat colour polymorphism in a free-living population of Soay sheep. Proc Biol Sci B 274: 619-626.

Jackson IJ, Chambers D, Rinchik EM, Bennett DC (1990). Characterization of TRP-1 mRNA levels in dominant and recessive mutations at the mouse brown $(b)$ locus. Genetics 126: $45-459$.

Jackson IJ (1994). Molecular and developmental genetics of mouse coat color. Annu Rev Genet 28: 189-217.

Javerzat S, Jackson IJ (1998). White-based brown (Tyrp1 $\left.{ }^{B-w}\right)$ is a dominant mutation causing reduced hair pigmentation owing to a chromosomal inversion. Mamm Genome 9: 469-471.

Johansson Moller M, Chaudhary R, Hellmen E, Hoyheim B, Chowdhary B, Andersson L (1996). Pigs with the dominant white coat color phenotype carry a duplication of the KIT gene encoding the mast/stem cell growth factor receptor. Mamm Genome 7: 822-830.

Kijas JM, Wales R, Tornsten A, Chardon P, Moller M, Andersson L (1998). Melanocortin receptor $1(M C 1 R)$ mutations and coat color in pigs. Genetics 150: 1177-1185.

Kijas JM, Moller M, Plastow G, Andersson L (2001). A frameshift mutation in $M C 1 R$ and a high frequency of somatic reversions cause black spotting in pigs. Genetics 158: 779-785.

Lai F, Ren J, Ai H, Ding N, Ma J, Zeng DQ et al. (2007). Chinese white Rongchang pig does not have the dominant white allele of KIT but has the dominant black allele of $M C 1 R$. J Hered 98: 84-87.
Lian LL, Wu ZD (1985). A study on the coat color inheritance of Dehe pig. Chinese J Anim Sci 21: 10-12.

Lyons LA, Foe IT, Rah HC, Grahn RA (2005). Chocolate coated cats: TYRP1 mutations for brown color in domestic cats. Mamm Genome 16: 356-366.

Mao HR, Ren J, Ding NS, Xiao SJ, Huang LS (2010). Genetic variation within coat color genes of $M C 1 R$ and ASIP in Chinese brownish red Tibetan pigs. Anim Sci J (e-pub ahead of print; doi:10.1111/j.1740-0929.2010.00789.x).

Marklund S, Kijas J, Rodriguez-Martinez H, Ronnstrand L, Funa K, Moller M et al. (1998). Molecular basis for the dominant white phenotype in the domestic pig. Genome Res 8: 826-833.

Nadeau NJ, Mundy NI, Gourichon D, Minvielle F (2007). Association of a single-nucleotide substitution in TYRP1 with roux in Japanese quail (Coturnix japonica). Anim Genet 38: 609-613.

Pielberg G, Olsson C, Syvanen AC, Andersson L (2002). Unexpectedly high allelic diversity at the KIT locus causing dominant white color in the domestic pig. Genetics 160: 305-311.

Ramos AM, Crooijmans RPMA, Affara NA, Amaral AJ, Archibald AL, Beever JE et al. (2009). Design of a high density SNP genotyping assay in the pig using SNPs identified and characterized by next generation sequencing technology. PLOS ONE 4: e6524.

Sarangarajan R, Boissy RE (2001). Tyrp1 and oculocutaneous albinism type 3. Pigment. Cell Res 14: 437-444.

Schmidt-Kuntzel A, Eizirik E, O'Brien SJ, Menotti-Raymond M (2005). Tyrosinase and tyrosinase related protein 1 alleles specify domestic cat coat color phenotypes of the albino and brown loci. J Hered 96: 289-301.

Schmutz SM, Berryere TG, Goldfinch AD (2002). TYRP1 and $M C 1 R$ genotypes and their effects on coat color in dogs. Mamm Genome 13: 380-387.

Searle AG (1968). Comparative Genetics of Coat Colour in Mammals. Academic Press: New York.

Silvers WK (1979). The Coat Colors of Mice: A Model for Mammalian Gene Action and Interaction. Springer-Verlag: New York.

Xu GL, Ren J, Ding NS, Ai HS, Guo YM, Chen CY et al. (2006). Genetic analysis of the KIT and MC1R genes in Chinese indigenous pigs with belt-like coat color phenotypes. Anim Genet 37: 518-519.

Zdarsky E, Favor J, Jackson IJ (1990). The molecular basis of brown, an old mouse mutation, and of an induced revertant to wild type. Genetics 126: 443-449.

Zhang ZG, Li BT, Chen XH (1986). Pig Breeds in China. Shanghai Scientific and Technical Publisher: Shanghai, China.

Supplementary Information accompanies the paper on Heredity website (http://www.nature.com/hdy) 